\title{
Universität des Saarlandes
}

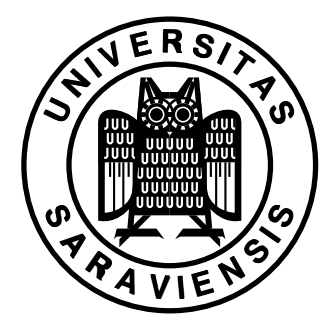

\section{Fachrichtung 6.1 - Mathematik}

Preprint

The behaviour of microstructures with small shears of the austenite-martensite interface in martensitic phase transformations

\author{
Martin Fuchs and Abdellah Elfanni
}

Preprint No. 34

Saarbrücken 2001 


\section{Universität des Saarlandes}

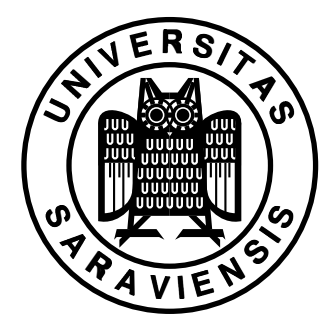

\section{Fachrichtung 6.1 - Mathematik}

\section{The behaviour of microstructures with small shears of the austenite-martensite interface in martensitic phase transformations}

\author{
Martin Fuchs \\ Saarland University \\ Department of Mathematics \\ Postfach 151150 \\ D-66041 Saarbrücken \\ Germany \\ E-Mail: fuchs@math.uni-sb.de
}

\author{
Abdellah Elfanni \\ Saarland University \\ Department of Mathematics \\ Postfach 151150 \\ D-66041 Saarbrücken \\ Germany \\ E-Mail: elfanni@math.uni-sb.de
}

submitted: 20th July 2001

Preprint No. 34

Saarbrücken 2001 
Edited by

FR 6.1 - Mathematik

Im Stadtwald

D-66041 Saarbrücken

Germany

Fax: $\quad+496813024443$

e-mail: preprint@math.uni-sb.de

WWW: http://www.math.uni-sb.de/ 


\begin{abstract}
Let $\Omega \subset \mathbb{R}^{2}$ denote a bounded domain whose boundary $\partial \Omega$ is Lipschitz and contains a segment $\Gamma_{0}$ representing the austenite-twinned martensite interface. We prove

$$
\inf _{u \in \mathcal{W}(\Omega)} \int_{\Omega} \varphi(\nabla u(x, y)) d x d y=0
$$

for any elastic energy density $\varphi: \mathbb{R}^{2} \rightarrow[0, \infty)$ such that $\varphi(0, \pm 1)=0$. Here $\mathcal{W}(\Omega)$ consists of all Lipschitz functions $u$ with $u=0$ on $\Gamma_{0}$ and $\left|u_{y}\right|=1$ a.e. Apart from the trivial case $\Gamma_{0} \subset \mathbb{R} \times\{a\}, a \in \mathbb{R}$, this result is obtained through the construction of suitable minimizing sequences which differ substantially for vertical and non-vertical segments.
\end{abstract}

AMS classification: 49,74

Keywords: microstructure, martensitic phase transformation, elastic energy, minimizing sequences, Young measures.

\title{
1 Introduction.
}

Let $\Omega$ be a bounded domain in $\mathbb{R}^{2}$ with a Lipschitz boundary $\partial \Omega$. We denote by $\Gamma_{0}$ a portion of the boundary $\partial \Omega$ defined as follows:

$$
\Gamma_{0}:=\left\{(x, y) \in \mathbb{R}^{2} \mid y=a x+b, \quad x \in[\alpha, \beta]\right\}
$$

or

$$
\Gamma_{0}:=\{a\} \times[\alpha, \beta]
$$

where $a, b, \alpha, \beta$ are real numbers. Let $\varphi: \mathbb{R}^{2} \rightarrow[0, \infty)$ denote a Borel function such that

$$
\varphi(0,1)=\varphi(0,-1)=0
$$

The class $\mathcal{W}$ of admissible comparison functions is introduced as follows

$$
\mathcal{W}:=\mathcal{W}(\Omega):=\left\{u \in W^{1, \infty}(\Omega):\left|u_{y}\right|=1 \text { a.e. in } \Omega \text { and } u=0 \text { on } \Gamma_{0}\right\} .(1
$$

Then we would like to consider the minimization problem

$$
I^{\infty}:=\inf _{u \in \mathcal{W}} \int_{\Omega} \varphi(\nabla u(x, y)) d x d y,
$$


more precisely, our goal is to calculate the number $I^{\infty}$ with the help of minimizing sequences. As a matter of fact (see for example Theorem 3 ) the existence of minimizers can in general not be expected. Problem (1.5) is of some physical interest. For instance, if we think of a model in martensitic phase transformation, $\varphi$ could be the elastic energy density of the martensite with two wells $(0,1)$ and $(0,-1)$ representing the stress-free states of two variants of the martensite. The portion $\Gamma_{0}$ of the boundary of $\Omega$ stands for the austenite-twinned martensite interface and the boundary condition $u=0$ on $\Gamma_{0}$ refers to elastic compatibility with the austenitic phase in the extreme case of complete rigidity of the austenite (see $\left[B . J_{1}\right],\left[B . J_{2}\right]$ and $[K o$.$] ).$

Problems of this type have been considered by M. Chipot and C. Collins but without the constraint $\left|u_{y}\right|=1$ a.e. ( see $[C$.$] and [C . C$.$] ). The con-$ straint $\left|u_{y}\right|=1$ is introduced in the paper $\left[\mathrm{K}_{\mathrm{M}} \mathrm{M}_{1}\right]$ of Kohn and Müller (see also $\left[\mathrm{K}_{\mathrm{M}} \mathrm{M}_{2}\right]$ ) where they discuss the behaviour of minimizing sequences for a functional consisting of elastic energy plus surface energy on suitable spaces. The main concern of our paper is to prove the following

Theorem : Under the above assumptions we have $I^{\infty}=0$.

For this purpose we will construct minimizing sequences which represent, according to the Ball-James theory, the microstructure and we will show that they differ substantially for domains with oblique interface $\Gamma_{0}$ and for domains having a vertical interface $\Gamma_{0}$. This is in contrast to the observation that both types of domains differ only by a simple geometric transformation.

Remark 1. When $\Gamma_{0}$ is parallel to the $x$-axis, i.e.

$$
\Gamma_{0}:=[\alpha, \beta] \times\{b\},
$$

then the quantity $I^{\infty}$ is easily seen to be equal to 0 . Indeed the function $u(x, y)=y-b$ belongs to $\mathcal{W}$ and $\nabla u(x, y)=(0,1)$ so that

$$
0 \leq I^{\infty} \leq \int_{\Omega} \varphi(\nabla u(x, y)) d x d y=0
$$

on account of (1.3).

Remark 2. It is sufficient to assume that the domain $\Omega$ is bounded with respect to the $y$ variable (see the proofs of Theorem 1. and Theorem 2 . below).

Remark 3. The functions forming minimizing sequences we will consider for domains having oblique austenite-martensite interfaces have a finite number of oscillations, hence they belong to the class

$$
\left\{u \in \mathcal{W}(\Omega):\left|u_{y y}\right| \text { is a Radon measure with finite mass }\right\}
$$


which was introduced by Winter [W.]. On the other hand the minimzing sequences we will construct for domains having vertical austenite-martensite interfaces have an infinite number of oscillations. This is an expected result due to Winter $[W$.] who proved for rectangular domains that functions in $\mathcal{W}$ have an infinite number of oscillations so that $\left|u_{y y}\right|$ cannot be a Radon measure with finite mass. In fact, the statement of [W.], Theorem 2.3, is misleading, he actually proved that his class $\mathcal{B}_{0}$ is empty.

Remark 4. The reader should note again that similar problems including a surface energy term but replacing the Lipschitz functions from our class $\mathcal{W}$ by functions from the space $W^{1,2}(\Omega)$ were already studied in the paper [K.M.1] of Kohn and Müller.

The paper is divided as follows. In section 2 we consider domains with oblique interfaces $\Gamma_{0}$ and construct a minimizing sequence of (1.5) with elastic energy going to zero. In section 3 we consider domains with vertical interfaces and also construct a minimizing sequence of (1.5). As already mentioned before the minimizing sequences are different for the two cases. In section 4 we study the nonexistence of minimizers for the problem (1.5) and show that the gradients of uniformly bounded minimizing sequences generate a unique Young measure supported by the wells $(0,1)$ and $(0,-1)$.

\section{Domains with an oblique austenite-martensite interface.}

In this section we assume that $\Gamma_{0}$ is oblique, i.e. $\Gamma_{0}$ is given by (1.1). Without loss of generality one can assume that $a \neq 0$, otherwise the infimum in (1.5) would be equal to zero and it is attained (see Remark 1.). Then we have the following theorem

Theorem 1. Let $\varphi: \mathbb{R}^{2} \rightarrow[0, \infty)$ be a Borel function such that (1.3) holds. Then

$$
I^{\infty}:=\inf _{u \in \mathcal{W}} \int_{\Omega} \varphi(\nabla u(x, y)) d x d y=0 .
$$

Proof. For notational simplicity we let $a=1$ and $b=0$, the general case will follow with obvious modifications. Given $\delta \in(0,1)$ we divide the square $(0, \delta) \times(0, \delta)$ as follows

$$
\begin{gathered}
\Delta_{0}=\left\{(x, y) \in \mathbb{R}^{2}: 0 \leq x \leq \delta, 0 \leq y \leq \frac{x}{2}\right\}, \\
P_{0}=\left\{(x, y) \in \mathbb{R}^{2}: 0 \leq x \leq \delta, \frac{x}{2} \leq y \leq \frac{x}{2}+\frac{\delta}{2}\right\},
\end{gathered}
$$




$$
\Delta_{1}=\left\{(x, y) \in \mathbb{R}^{2}: 0 \leq x \leq \delta, \frac{x}{2}+\frac{\delta}{2} \leq y \leq \delta\right\}
$$

and define a function $u: S_{0}:=\mathbb{R} \times[0, \delta] \rightarrow \mathbb{R}$ as follows

$$
u(x, y)= \begin{cases}y \quad \text { if } \quad(x, y) \in \Delta_{0} \cup(\delta, \infty) \times\left(0, \frac{\delta}{2}\right), \\ x-y \quad \text { if } \quad(x, y) \in P_{0}, \\ \delta-y \quad \text { if } \quad(x, y) \in(\delta, \infty) \times\left(\frac{\delta}{2}, \delta\right), \\ y-\delta \quad \text { if } \quad(x, y) \in \Delta_{1} \cup(-\infty, 0) \times\left(\frac{\delta}{2}, \delta\right), \\ -y \quad \text { if } \quad x \leq 0,0 \leq y \leq \frac{\delta}{2} .\end{cases}
$$

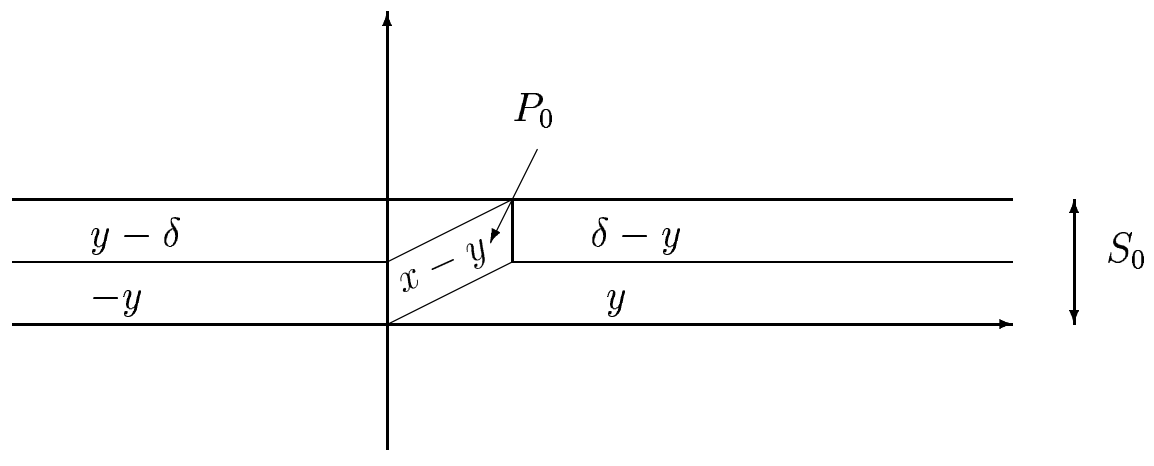

The function $u$ is a Lipschitz function vanishing on $\mathbb{R} \times\{0\}$ and $\mathbb{R} \times\{\delta\}$. Let

$$
S_{k}=\mathbb{R} \times[\delta k, \delta(k+1)], k \in \mathbb{Z},
$$

and define $u: \mathbb{R}^{2} \rightarrow \mathbb{R}$ via

$$
u(x, y)=u(x-k \delta, y-k \delta) \quad \text { if } \quad(x, y) \in S_{k} .
$$

¿From our construction we deduce

$$
\left|u_{y}\right|=1 \quad \text { a.e. in } \mathbb{R}^{2} \text {. }
$$

$u$ also satisfies

$$
\left|u_{x}\right|=0 \quad \text { a.e. in } \mathbb{R}^{2} \backslash \bigcup_{k=-\infty}^{\infty} P_{k}
$$


where $P_{k}, k \neq 0$, has an obvious meaning. The Lebesgue measure of $P_{k}$ is

$$
\left|P_{k}\right|=\frac{\delta^{2}}{2}
$$

Note also that $u$ vanishes on the diagonal $x=y$. Now the proof of Theorem 1 can be finished easily. Since $\varphi$ is nonnegative and since the restriction of $u$ to $\Omega$ belongs to $\mathcal{W}$ one has

$$
0 \leq I^{\infty} \leq \int_{\Omega} \varphi(\nabla u(x, y)) d x d y
$$

Let us denote by $N:=N(\delta)$ the smallest number of strips $S_{k}, k \in \mathbb{Z}$, that are sufficient to cover the domain $\Omega$. Therefore there exists $p \in \mathbb{Z}$ such that

$$
\int_{\Omega} \varphi(\nabla u(x, y)) d x d y \leq \sum_{k=p}^{p+N-1} \int_{\Omega \cap S_{k}} \varphi(\nabla u(x, y)) d x d y .
$$

Combining (2.1), (2.2) and (1.3) one has for every $k \in \mathbb{Z}$

$$
\int_{\Omega \cap S_{k}} \varphi(\nabla u(x, y)) d x d y=\int_{\Omega \cap P_{k}} \varphi(\nabla u(x, y)) d x d y .
$$

Observing

$$
\nabla u(x, y)=(1,-1) \text { on } P_{k}
$$

we deduce

$$
\begin{array}{r}
\int_{\Omega \cap P_{k}} \varphi\left(\nabla u_{k}(x, y)\right) d x d y=\varphi(1,-1)\left|\Omega \cap P_{k}\right| \\
\leq \varphi(1,-1)\left|P_{k}\right|
\end{array}
$$

Combining (2.5), (2.6) and (2.3) we get the estimate

$$
\int_{\Omega} \varphi(\nabla u(x, y)) d x d y \leq N \varphi(1,-1) \frac{\delta^{2}}{2} .
$$

Note that

$$
(N-1) \delta \leq \operatorname{diam}(\Omega)
$$

where $\operatorname{diam}(\Omega)$ denotes the diameter of $\Omega$. Since $\delta \in(0,1)$ one gets

$$
N \delta \leq \operatorname{diam}(\Omega)+1
$$

Using (2.4)-(2.7) and (2.8) we obtain the final estimate

$$
0 \leq I^{\infty} \leq \frac{\delta}{2}(\operatorname{diam}(\Omega)+1) \varphi(1,-1)
$$


and since $\delta \in(0,1)$ is arbitrary one concludes that

$$
I^{\infty}=0
$$

\section{Domains with vertical austenite-martensite interfaces.}

In this section we assume that $\Gamma_{0}$ is vertical, i.e. $\Gamma_{0}$ is defined by (1.2). Without loss of generality we let $a=0$. Indeed, if $a$ is different from 0 , let us denote by $\Omega_{a}$ the following set

$$
\Omega_{a}=\{(x-a, y) \mid(x, y) \in \Omega\} .
$$

Then

$$
u \in \mathcal{W}(\Omega) \Longleftrightarrow v \in \mathcal{W}\left(\Omega_{a}\right)
$$

where $v$ is defined in $\Omega_{a}$ by

$$
v(x, y)=u(x+a, y)
$$

Obviously we have

$$
\int_{\Omega} \varphi(\nabla u(x, y)) d x d y=\int_{\Omega_{a}} \varphi(\nabla v(x, y)) d x d y
$$

therefore

$$
\inf _{u \in \mathcal{W}(\Omega)} \int_{\Omega} \varphi(\nabla u(x, y)) d x d y=\inf _{v \in \mathcal{W}\left(\Omega_{a}\right)} \int_{\Omega_{a}} \varphi(\nabla v(x, y)) d x d y
$$

Now we have:

Theorem 2. Consider any Borel function $\varphi: \mathbb{R}^{2} \rightarrow[0, \infty)$. Then, if (1.2) and (1.3) hold, one has

$$
I^{\infty}:=\inf _{u \in \mathcal{W}(\Omega)} \int_{\Omega} \varphi(\nabla u(x, y)) d x d y=0 .
$$


In order to prove Theorem 2. we will need some preparatory lemmas in which we slightly refine the construction of Kohn and Müller (see $\left[K . M_{1}\right]$ ). First we define a function $\nu:[0,1] \times\left[0, \frac{1}{2}\right] \rightarrow \mathbb{R}$ by

$$
\nu(x, y):=\left\{\begin{array}{lll}
y, & \text { if } & 0 \leq y \leq \frac{x+1}{8} \\
\frac{x+1}{4}-y, & \text { if } & \frac{x+1}{8} \leq y \leq \frac{x+3}{8} \\
y-\frac{1}{2}, & \text { if } & \frac{x+3}{8} \leq y \leq \frac{1}{2} .
\end{array}\right.
$$

Then we extend $\nu$ to $[0,1] \times[0,1]$ antiperiodically by letting

$$
\bar{\nu}(x, y):= \begin{cases}\nu(x, y), & \text { if } \quad(x, y) \in[0,1] \times\left[0, \frac{1}{2}\right] \\ -\nu(x, 1-y), & \text { if } \quad(x, y) \in[0,1] \times\left[\frac{1}{2}, 1\right] .\end{cases}
$$

Note that $\bar{\nu}$ is a Lipschitz function such that

$$
\begin{array}{r}
\left|\bar{\nu}_{y}\right|=1 \text { a.e. } \\
\bar{\nu}(x, 0)=\bar{\nu}(x, 1)=0,
\end{array}
$$

and

$$
\bar{\nu}_{x}(x, y)=0 \text { if }(x, y) \notin S,
$$

the set $S:=S_{1} \cup S_{2}$ being defined as follows

$$
\begin{aligned}
& S_{1}:=\left\{(x, y) \in[0,1] \times[0,1] \mid \frac{x+1}{8} \leq y \leq \frac{x+3}{8}\right\}, \\
& S_{2}:=\left\{(x, y) \in[0,1] \times[0,1] \mid \frac{5-x}{8} \leq y \leq \frac{7-x}{8}\right\} .
\end{aligned}
$$

Obviously

$$
\begin{gathered}
\nabla \bar{\nu}(x, y)=\left(\frac{1}{4},-1\right) \text { in } S_{1}, \\
\nabla \bar{\nu}(x, y)=\left(-\frac{1}{4},-1\right) \text { in } S_{2},
\end{gathered}
$$


and using (1.3) and (3.1)-(3.7) we get

$$
\int_{0}^{1} \int_{0}^{1} \varphi(\nabla \bar{\nu}(x, y)) d x d y=\int_{S_{1}} \varphi(\nabla \bar{\nu}(x, y)) d x d y+\int_{S_{2}} \varphi(\nabla \bar{\nu}(x, y)) d x d y
$$

that is

$$
\int_{0}^{1} \int_{0}^{1} \varphi(\nabla \bar{\nu}(x, y)) d x d y=\frac{1}{4}\left(\varphi\left(\frac{1}{4},-1\right)+\varphi\left(-\frac{1}{4},-1\right)\right) .
$$

Due to (3.2) we may extend $\bar{\nu}$ periodically with respect to $y$ by letting

$$
\bar{\nu}(x, y+1)=\bar{\nu}(x, y), \quad(x, y) \in[0,1] \times \mathbb{R} .
$$

Notice that $\bar{\nu}$ also satisfies

$$
\bar{\nu}(0, y)=\frac{1}{2} \bar{\nu}(1,2 y) .
$$

Now let us define the function $w:\left[\frac{1}{2}, 1\right] \times \mathbb{R} \rightarrow \mathbb{R}$ as follows

$$
w(x, y)=\bar{\nu}(2 x-1, y) .
$$

We finally let $u:[0,1] \times \mathbb{R} \rightarrow \mathbb{R}$,

$$
u(x, y)=\left\{\begin{array}{l}
\left.\left.\sum_{i=0}^{+\infty} \frac{1}{2^{i}} w\left(2^{i} x, 2^{i} y\right) \chi_{\left[\frac{1}{2^{i}+1}, \frac{1}{2^{i}}\right]}(x) \quad \text { if } \quad x \in\right] 0,1\right] \\
0 \quad \text { if } \quad x=0,
\end{array}\right.
$$

where $\chi_{\left[\frac{1}{2^{i}+1}, \frac{1}{2^{i}}\right]}$ denotes the characteristic function of the interval $\left[\frac{1}{2^{i+1}}, \frac{1}{2^{i}}\right]$. Then we have the following lemma

Lemma 1. Let $u$ be the function defined by (3.11). Then $u$ is a Lipschitz continuous function such that

$$
\begin{array}{r}
\left|u_{y}\right|=1 \text { a.e. in }[0,1] \times \mathbb{R}, \\
u(x, 0)=u(x, 1)=0 \text { for all } x \in[0,1] .
\end{array}
$$

Proof. Due to (3.9) the functions

$$
\sum_{i=0}^{k} \frac{1}{2^{i}} w\left(2^{i} x, 2^{i} y\right) \chi_{\left[\frac{1}{2^{i}+1}, \frac{1}{2^{i}}\right]}(x)
$$


are continuous and converge uniformly to $u$ in $] 0,1] \times \mathbb{R}$. Therefore $u$ is continuous in $] 0,1] \times \mathbb{R}$. Since the function $w$ is bounded and

$$
\lim _{x \rightarrow 0} \chi_{\left[\frac{1}{2^{i+1}}, \frac{1}{2^{i}}\right]}(x)=0,
$$

we get

$$
\lim _{x \rightarrow 0} u(x, y)=0 .
$$

Thus $u$ is a continuous function on $[0,1] \times \mathbb{R}$. Now let $(x, y) \in] 0,1] \times \mathbb{R}$. Then there exists $i \in \mathbb{N}$ such that

$$
x \in\left[\frac{1}{2^{i+1}}, \frac{1}{2^{i}}\right]
$$

and therefore

$$
u(x, y)=\frac{1}{2^{i}} w\left(2^{i} x, 2^{i} y\right)
$$

Combining (3.1) and (3.10) one easily gets (3.12). Using (3.13) and (3.10) we see that

$$
u(x, 0)=\frac{1}{2^{i}} w\left(2^{i} x, 0\right)=\frac{1}{2^{i}} \bar{\nu}\left(2^{i+1} x-1,0\right),
$$

hence

$$
u(x, 0)=0
$$

on account of (3.2). On the other hand we have

$$
u(x, 1)=\frac{1}{2^{i}} \bar{\nu}\left(2^{i+1} x-1,2^{i}\right)
$$

and since $\bar{\nu}$ is 1 -periodic with respect to $y$, this implies by $(3.2)$

$$
u(x, 1)=\frac{1}{2^{i}} \bar{\nu}\left(2^{i+1} x-1,1\right)=0 .
$$

Lemma 2. Let $u$ be the function defined by (3.11). Under the above assumptions we have

$$
\int_{0}^{1} \int_{0}^{1} \varphi(\nabla u(x, y)) d x d y=\frac{1}{4}\left[\varphi\left(\frac{1}{2},-1\right)+\varphi\left(-\frac{1}{2},-1\right)\right] .
$$

Proof. First, let us calculate 


$$
I_{i}:=\int_{\frac{1}{2^{i+1}}}^{\frac{1}{2^{i}}} d x \int_{0}^{1} d y \varphi(\nabla u(x, y)), \quad i \in \mathbb{N} .
$$

By definition we have

$$
I_{i}=\int_{\frac{1}{2^{i+1}}}^{\frac{1}{2^{i}}} d x \int_{0}^{1} d y \varphi\left(w_{x}\left(2^{i} x, 2^{i} y\right), w_{y}\left(2^{i} x, 2^{i} y\right)\right)
$$

and using (3.10) we get

$$
I_{i}=\int_{\frac{1}{2^{i+1}}}^{\frac{1}{2^{i}}} d x \int_{0}^{1} d y \varphi\left(2 \bar{\nu}_{x}\left(2^{i+1} x-1,2^{i} y\right), \bar{\nu}_{y}\left(2^{i+1} x-1,2^{i} y\right)\right)
$$

By a change of variables we get

$$
I_{i}=\frac{1}{2^{2 i+1}} \int_{0}^{1} d x \int_{0}^{2^{i}} d y \varphi\left(2 \bar{\nu}_{x}(x, y), \bar{\nu}_{y}(x, y)\right)
$$

and the periodicity of $\bar{\nu}$ wih respect to y implies

$$
I_{i}=\frac{1}{2^{i+1}} \int_{0}^{1} \int_{0}^{1} \varphi\left(2 \bar{\nu}_{x}(x, y), \bar{\nu}_{y}(x, y)\right) d x d y
$$

so that

$$
I_{i}=\frac{1}{2^{i+1}} \int_{0}^{1} \int_{0}^{1} \tilde{\varphi}(\nabla \bar{\nu}(x, y)) d x d y
$$

where

$$
\tilde{\varphi}\left(\lambda_{1}, \lambda_{2}\right)=\varphi\left(2 \lambda_{1}, \lambda_{2}\right) .
$$

Applying (3.8) to $\tilde{\varphi}$ we get

$$
\int_{0}^{1} \int_{0}^{1} \tilde{\varphi}(\nabla \bar{\nu}(x, y)) d x d y=\frac{1}{4}\left[\tilde{\varphi}\left(\frac{1}{4},-1\right)+\tilde{\varphi}\left(-\frac{1}{4},-1\right)\right]
$$

thus

$$
\int_{0}^{1} \int_{0}^{1} \tilde{\varphi}(\nabla \bar{\nu}(x, y)) d x d y=\frac{1}{4}\left[\varphi\left(\frac{1}{2},-1\right)+\varphi\left(-\frac{1}{2},-1\right)\right] .
$$

Therefore we arrive at

$$
I_{i}=\frac{1}{2^{i+3}}\left[\varphi\left(\frac{1}{2},-1\right)+\varphi\left(-\frac{1}{2},-1\right)\right],
$$

and since

$$
\int_{0}^{1} \int_{0}^{1} \varphi(\nabla u(x, y)) d x d y=\sum_{i=0}^{+\infty} I_{i}
$$


we finally deduce

$$
\int_{0}^{1} \int_{0}^{1} \varphi(\nabla u(x, y)) d x d y=\frac{1}{4}\left[\varphi\left(\frac{1}{2},-1\right)+\varphi\left(-\frac{1}{2},-1\right)\right]
$$

which completes the proof of the lemma.

Proof of Theorem 2. Let $\delta \in(0,1)$ and define $v:[0, \delta] \times \mathbb{R} \rightarrow \mathbb{R}$ by rescaling the function $u$ from (3.11), i.e.

$$
v(x, y)=\delta u\left(\frac{x}{\delta}, \frac{y-k \delta}{\delta}\right) \quad \text { if } \quad(x, y) \in[0, \delta] \times[k \delta,(k+1) \delta],
$$

where $k \in \mathbb{Z}$. The function $v$ is extended to $[0,+\infty[\times \mathbb{R}$ via

$$
v(x, y)=v(\delta, y) \quad \text { if } \quad(x, y) \in[\delta,+\infty[\times \mathbb{R} .
$$

Notice that $v$ is a continuous function vanishing for $x=0$. We can further extend $v$ to a continuous function defined in $\mathbb{R}^{2}$ by reflection :

$$
v(x, y)=v(-x, y) \quad \text { if } \quad(x, y) \in]-\infty, 0[\times \mathbb{R} .
$$

It is clear that $v$ is a Lipschitz function such that

$$
\begin{array}{r}
\left|v_{y}\right|=1 \quad \text { a.e. in } \quad \mathbb{R}^{2}, \\
v_{x}=0 \quad \text { if } \quad|x| \geq \delta .
\end{array}
$$

We denote by $\left(R_{k}\right)_{k \in \mathbb{Z}}$ and $\left(S_{k}\right)_{k \in \mathbb{Z}}$ the following rectangles and strips

$$
R_{k}=[-\delta, \delta] \times[k \delta,(k+1) \delta], S_{k}=\mathbb{R} \times[k \delta,(k+1) \delta] .
$$

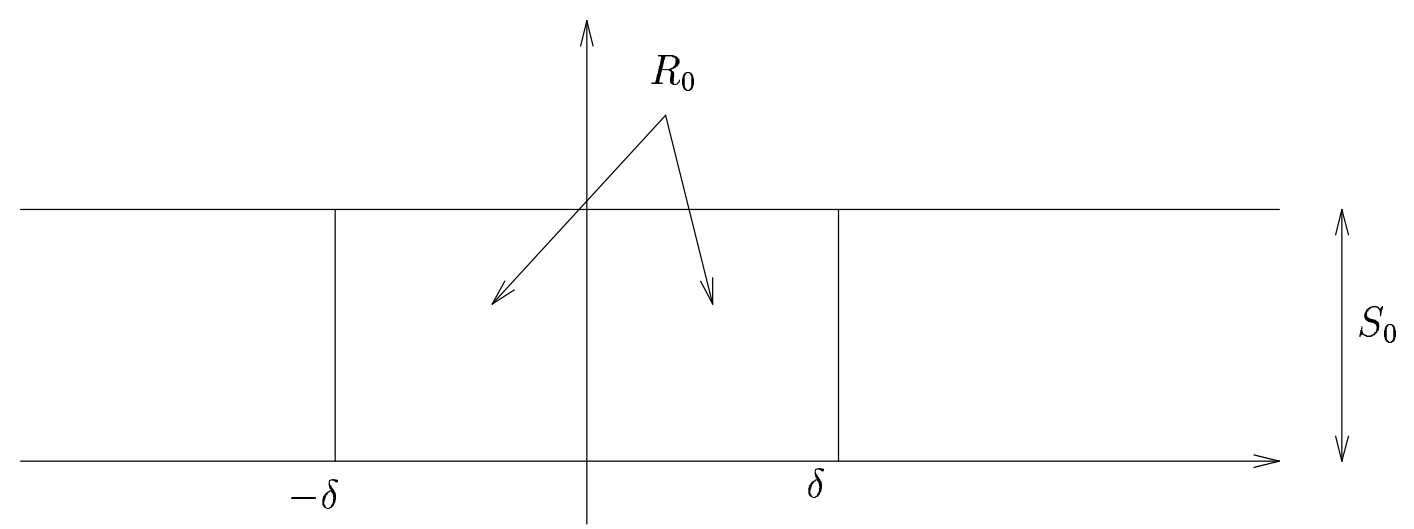


Let $N=N(\delta)$ denote the minimal number of strips which are sufficient to cover the domain $\Omega$. Since the restriction of $v$ to $\Omega$ belongs to $\mathcal{W}$, one has

$$
I^{\infty} \leq \int_{\Omega} \varphi(\nabla v(x, y)) d x d y
$$

There exists $p \in \mathbb{Z}$ such that

$$
\int_{\Omega} \varphi(\nabla v(x, y)) d x d y=\sum_{k=p}^{p+N-1} \int_{\Omega \cap S_{k}} \varphi(\nabla v(x, y)) d x d y
$$

Using (1.3), (3.15) and (3.16) we get for $k \in\{p, p+1, \ldots,(p+N-1)\}$

$$
\int_{\Omega \cap S_{k}} \varphi(\nabla v(x, y)) d x d y=\int_{\Omega \cap R_{k}} \varphi(\nabla v(x, y)) d x d y
$$

so that

$$
\int_{\Omega} \varphi(\nabla v(x, y)) d x d y \leq \sum_{k=p}^{p+N-1} \int_{R_{k}} \varphi(\nabla v(x, y)) d x d y
$$

Obviously

$$
\int_{R_{k}} \varphi(\nabla v(x, y)) d x d y=I_{1}+I_{2}
$$

where $I_{1}$ and $I_{2}$ denote the following quantities

$$
I_{1}:=\int_{0}^{\delta} \int_{k \delta}^{(k+1) \delta} \varphi(\nabla v(x, y)) d x d y, I_{2}:=\int_{-\delta}^{0} \int_{k \delta}^{(k+1) \delta} \varphi(\nabla v(x, y)) d x d y
$$

From (3.14) we deduce

$$
I_{1}=\int_{0}^{\delta} d x \int_{k \delta}^{(k+1) \delta} d y \varphi(\nabla v(x, y))=\int_{0}^{\delta} d x \int_{k \delta}^{(k+1) \delta} d y \varphi\left(\nabla u\left(\frac{x}{\delta}, \frac{y-k \delta}{\delta}\right)\right),
$$

and after a change of variables we arrive at

$$
I_{1}=\delta^{2} \int_{0}^{1} \int_{0}^{1} \varphi(\nabla u(x, y)) d x d y
$$

According to Lemma 2. we get

$$
I_{1}=\frac{\delta^{2}}{4}\left[\varphi\left(\frac{1}{2},-1\right)+\varphi\left(-\frac{1}{2},-1\right)\right] .
$$


In a similar way we deduce

$$
I_{2}=\frac{\delta^{2}}{4}\left[\varphi\left(\frac{1}{2},-1\right)+\varphi\left(-\frac{1}{2},-1\right)\right]
$$

and therefore

$$
\int_{R_{k}} \varphi(\nabla u(x, y)) d x d y=\frac{\delta^{2}}{2}\left[\varphi\left(\frac{1}{2},-1\right)+\varphi\left(-\frac{1}{2},-1\right)\right] .
$$

Using (3.17) we get the bound

$$
\int_{\Omega} \varphi(\nabla v(x, y)) d x d y \leq N \frac{\delta^{2}}{2}\left[\varphi\left(\frac{1}{2},-1\right)+\varphi\left(-\frac{1}{2},-1\right)\right] .
$$

and as in section 2 we obtain

$$
0 \leq I^{\infty} \leq(\operatorname{diam}(\Omega)+1) \frac{\delta}{2}\left[\varphi\left(\frac{1}{2},-1\right)+\varphi\left(-\frac{1}{2},-1\right)\right],
$$

thus

$$
I^{\infty}=0
$$

\section{Nonexistence of minimizers and Young Mea- sures}

In this section we assume that $\varphi$ satisfies in addition to (1.3)

$$
\varphi\left(\lambda_{1}, \lambda_{2}\right)=0 \text { if and only if }\left(\lambda_{1}, \lambda_{2}\right)=(0, \pm 1) .
$$

We have the following Poincaré type inequality Lemma 3. Consider a domain $\Omega$ as in section 1. Then, for any function $u \in W^{1, \infty}(\Omega)$ such that $u=0$ on $\Gamma_{0}$, we have

$$
\int_{\Omega_{0}}|u(x, y)| d x d y \leq C\left(\Omega_{0}\right) \int_{\Omega_{0}}\left|u_{x}(x, y)\right| d x d y
$$

where $C\left(\Omega_{0}\right)$ is a constant which only depends on $\Omega_{0}$. In case (1.2) $\Omega_{0}$ can denote any rectangle in $\Omega$ with one vertical boundary part contained in $\Gamma_{0}$, in case (1.1) we can choose an appropriate parallelogram or triangle. 


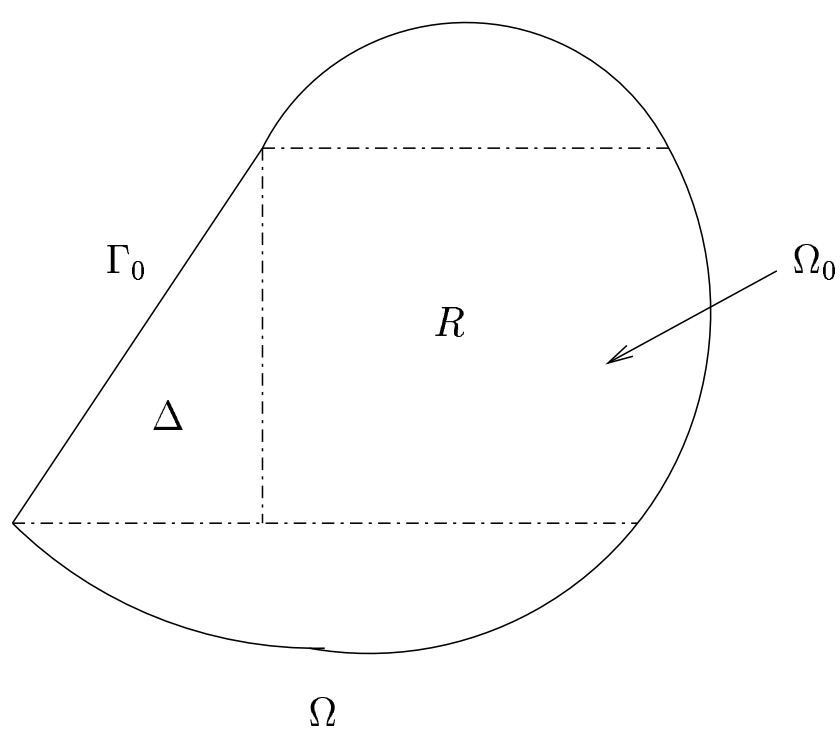

Proof. Let us first consider the case where $\Gamma_{0}$ is vertical, i.e. (1.2) holds. Then there exists $(b, \varepsilon) \in \mathbb{R} \times \mathbb{R}_{+}$such that

$$
(a, a+\varepsilon) \times(b-\varepsilon, b+\varepsilon) \subset \Omega \text { or }(a-\varepsilon, a) \times(b-\varepsilon, b+\varepsilon) \subset \Omega
$$

and

$$
\{a\} \times(b-\varepsilon, b+\varepsilon) \subset \Gamma_{0} .
$$

Assume that we have

$$
(a, a+\varepsilon) \times(b-\varepsilon, b+\varepsilon) \subset \Omega,
$$

and set for simplicity

$$
\Omega_{0}:=(a, a+\varepsilon) \times(b-\varepsilon, b+\varepsilon) .
$$

The general case can be handled similarly. Let $(x, y) \in \Omega_{0}$ and consider $u \in \mathcal{W}(\Omega)$ such that $u=0$ on $\Gamma_{0}$. Then

$$
u(x, y)=u(x, y)-u(a, y)=\int_{a}^{x} u_{x}(t, y) d t
$$

so that

$$
|u(x, y)| \leq \int_{a}^{x}\left|u_{x}(t, y)\right| d t \leq \int_{a}^{a+\varepsilon}\left|u_{x}(t, y)\right| d t .
$$

and in conclusion

$$
\int_{a}^{a+\varepsilon}|u(x, y)| d x \leq \varepsilon \int_{a}^{a+\varepsilon}\left|u_{x}(t, y)\right| d t
$$


By integrating with respect to $y$ we obtain (4.2). Now consider the case where $\Gamma_{0}$ is defined by (1.1). Without loss of generality we may assume that $a$ is positive and that the triangle

$$
\Delta:=\left\{(x, y): x_{0}<x<x_{0}+\varepsilon, a x_{0}+b<y<a x+b\right\}
$$

belongs to $\Omega$ with boundary part $\left\{(x, y): x_{0} \leq x \leq x_{0}+\varepsilon, y=a x+b\right\}$ contained in $\Gamma_{0}$. Let $(x, y) \in \Delta$ and choose $u \in W^{1, \infty}(\Omega)$ such that $u=0$ on $\Gamma_{0}$. Then

$$
u(x, y)=u(x, y)-u\left(\frac{y-b}{a}, y\right)=\int_{\frac{y-b}{a}}^{x} u_{x}(t, y) d t
$$

and therefore

$$
|u(x, y)| \leq \int_{\frac{y-b}{a}}^{x}\left|u_{x}(t, y)\right| d t
$$

This gives

$$
\int_{\frac{y-b}{a}}^{x_{0}+\epsilon}|u(x, y)| d x \leq\left(x_{0}+\varepsilon-\frac{y-b}{a}\right) \int_{\frac{y-b}{a}}^{x_{0}+\epsilon}\left|u_{x}(t, y)\right| d t \leq \varepsilon \int_{\frac{y-b}{a}}^{x_{0}+\epsilon}\left|u_{x}(t, y)\right| d t
$$

and after integration with respect to $y$ we get (4.2) for the triangle. Clearly the same argument can be applied to the region $\Omega_{0}=\Delta \cup R$ where $R$ denotes some domain as in the figure above.

Lemma 3 implies the following theorem

Theorem 3. Assume that $\varphi: \mathbb{R}^{2} \rightarrow[0, \infty)$ is a Borel function such that (1.3) and the following weaker version of (4.1) hold

$$
\varphi\left(\lambda_{1}, \lambda_{2}\right)=0 \Longrightarrow \lambda_{1}=0
$$

Then the problem

$$
I^{\infty}:=\inf _{v \in \mathcal{W}} \int_{\Omega} \varphi(\nabla v(x, y)) d x d y
$$

cannot attain its infimum.

Proof. Let $u \in \mathcal{W}(\Omega)$ such that

$$
\int_{\Omega} \varphi(\nabla u(x, y)) d x d y=0 .
$$

¿From (4.1)’ together with $u \in \mathcal{W}(\Omega)$ we deduce

$$
\nabla u(x, y)=(0, \pm 1) \text { a.e. in } \Omega \text {, }
$$


and Lemma 3 implies that $u(x, y)=0$ on an appropriate subdomain $\Omega_{0}$ of $\Omega$. This contradicts the fact that

$$
\left|u_{y}\right|=1 \text { a.e. in } \Omega \text {. }
$$

Therefore the problem (4.3) cannot attain its infimum.

In the sequel we will study the behaviour of minimizing sequences of the problem (4.3) using the theory of Young measures.

Theorem 4. Let $\Omega$ denote a domain as in Section 1 with the additional property that (4.2) is true with the choice $\Omega_{0}=\Omega$. Assume that the function $\varphi: \mathbb{R}^{2} \rightarrow[0, \infty)$ is continuous and satisfies (4.1). Let $\left(u_{n}\right)_{n}$ be a minimizing sequence of the problem (4.3) such that

$$
\left|u_{n}\right|_{\infty},\left|\left(u_{n}\right)_{x}\right|_{\infty} \leq C
$$

where $C$ is a constant independent of $n$. Then

$$
u_{n} \rightarrow 0 \text { uniformly in } \Omega \text {. }
$$

Moreover, the sequence of gradients $\left(\nabla u_{n}\right)_{n}$ defines a Young measure $\left(\nu_{X}\right)_{X \in \Omega}$ on $\mathbb{R}^{2}$ which is given by

$$
\nu_{X}=\frac{1}{2} \delta_{(0,-1)}+\frac{1}{2} \delta_{(0,1)} \text { a.e. in } \Omega
$$

where $\delta_{w}$ is the Dirac mass at the point $w$.

Proof. By (4.4) there exist $u \in W^{1, \infty}(\Omega)$ and a subsequence of $\left(u_{n}\right)_{n}$ that we also denote by $\left(u_{n}\right)_{n}$, such that

$$
u_{n} \rightarrow u \text { uniformly in } \Omega
$$

and

$$
\nabla u_{n} \rightarrow \nabla u \text { in } L^{\infty}(\Omega)^{2} \text { weak - * . }
$$

Now the bounded sequence of gradients generates a Young measure on $\mathbb{R}^{2}$ (see [P.]) in the sense that there is a probability measure $\nu_{X}$ on $\mathbb{R}^{2}$ and a subsequence of $\nabla u_{n}$ such that for any Carathéodory function $\mathrm{F}$ on $\Omega \times \mathbb{R}^{2}$ one has

$$
F\left(X, \nabla u_{n}\right) \rightarrow \int_{\mathbb{R}^{2}} F(X, \lambda) d \nu_{X}(\lambda) \text { in } L^{\infty}(\Omega)^{2} \text { weak - * }
$$


Using (4.7) with $F=\varphi$ and exploiting the fact that $\left(u_{n}\right)_{n}$ is a minimizing sequence one gets

$$
\int_{\Omega} \varphi\left(\nabla u_{n}\right) d X \rightarrow 0=\int_{\Omega} \int_{\mathbb{R}^{2}} \varphi(\lambda) d \nu_{X}(\lambda) d X
$$

It follows that

$$
\int_{\mathbb{R}^{2}} \varphi(\lambda) d \nu_{X}(\lambda)=0 \text { a.e. in } \Omega
$$

hence

$$
\operatorname{Supp}\left(\nu_{X}\right) \subset\{(0, \pm 1)\} \text { for a.e. } X \in \Omega \text {, }
$$

where $\operatorname{Supp}\left(\nu_{X}\right)$ denotes the support of $\nu_{X}$. Therefore there exists a measurable function $\alpha$ such that

$$
0 \leq \alpha(X) \leq 1 \text { for a.e. } X \in \Omega
$$

and

$$
\nu_{X}=\alpha(X) \delta_{(0,-1)}+(1-\alpha(X)) \delta_{(0,1)} .
$$

The choice $F\left(X,\left(\lambda_{1}, \lambda_{2}\right)\right)=\left|\lambda_{1}\right|$ implies

$$
\int_{\Omega}\left|\left(u_{n}\right)_{x}\right| d x d y \rightarrow \int_{\Omega} \int_{\mathbb{R}^{2}}\left|\lambda_{1}\right| d \nu_{X} d x d y=0
$$

the last equality being a consequence of (4.8). On the other hand by lower semicontinuity we have

$$
\liminf _{n} \int_{\Omega}\left|\left(u_{n}\right)_{x}\right| d x d y \geq \int_{\Omega}\left|u_{x}\right| d x d y
$$

so that

$$
\int_{\Omega}\left|u_{x}\right| d x d y=0
$$

Now our assumption concerning $\Omega$ gives $u=0$. Since 0 is the unique limit point of $\left(u_{n}\right)_{n}$ one obtains (4.5). Moreover, for any disc $D \subset \Omega$

$$
\nabla u_{n} \rightarrow u \text { in } L^{\infty}(D)^{2} \text { weak -* }
$$

implies

$$
\int_{D}\left(u_{n}\right)_{y} d x d y \rightarrow 0
$$


therefore (consider $F\left(X,\left(\lambda_{1}, \lambda_{2}\right)\right)=\chi_{D}(X) \lambda_{2}$ in $(4.7)$ )

$$
\int_{D} \int_{\mathbb{R}^{2}} \lambda_{2} d \nu_{X}(\lambda) d X=0
$$

From (4.9) we deduce

$$
\int_{D}(1-2 \alpha(X)) d x d y=0
$$

and since $D$ is arbitrary, we get $\alpha(x)=\frac{1}{2}$ a.e. This proves (4.6).

Remark 5. Our assumptions on $\Omega$ are true if we consider a rectangle or a parallelogram and if $\Gamma_{0}$ is just one of non-horizontal boundary parts of $\Omega$.

Remark 6. If we want to have the convergence stated in (4.5), then we are forced to consider the situation described in Remark 5. For example, let

$$
\Omega:=[0,1] \times[0,1+\varepsilon], \Gamma_{0}:=\{0\} \times[0,1] .
$$

With $\delta=\frac{1}{m}, m \in \mathbb{N}$, we define $v_{m}$ according to $(3.14)$ on $[0, \delta] \times[0,1]$. On $[\delta, 1] \times[0,1]$ we let

$$
v_{m}(x, y)=v_{m}(\delta, y)
$$

and on $[0,1] \times[1,1+\varepsilon]$ we define

$$
v_{m}(x, y)=y-1
$$

which is a function of class $\mathcal{W}(\Omega)$. Clearly

$$
\int_{\Omega} \varphi\left(\nabla v_{m}(x, y)\right) d x d y=\int_{0}^{1} \int_{0}^{1} \varphi\left(\nabla v_{m}(x, y)\right) d x d y \rightarrow 0
$$

when $m$ goes to infinity. Thus the sequence $\left(v_{m}\right)_{m}$ is a minimizing sequence which does not converge uniformly to zero on the whole domain $\Omega$. Note also that the sequence of gradients of $v_{m}$ generates a Young measure $\left(\mu_{X}\right)_{X \in \Omega}$ such that

$$
\mu_{X}=\frac{1}{2} \delta_{(0,-1)}+\frac{1}{2} \delta_{(0,1)} \text { for a.e. } X \in[0,1] \times[0,1],
$$

and

$$
\mu_{X}=\delta_{(0,1)} \text { for a.e. } X \in[0,1] \times[1,1+\varepsilon] .
$$

If we let $v_{m}(x, y)=1-y$ in (4.10) we obtain again a minimizing sequence such that

$$
\mu_{X}=\delta_{(0,-1)} \text { for a.e. } X \in[0,1] \times[1,1+\varepsilon]
$$

and therefore we have no uniqueness for the Young measure. 


\section{References}

[B.J.1] J. M. Ball and R. D. James : Fine phase mixtures as minimizers of energy. Arch. Rational Mech. Anal., 100, (1987), p. 13-52.

[B.J.2] J. M. Ball and R. D. James : Proposed experimental tests of a theory of fine microstructures. Phil. Trans. Roy. Soc. London A, 338, (1992), p. 350-389.

[B.M] J. M. Ball and F. Murat : $W^{1, p}$ quasiconvexity and variational problems for multiple integrals. J. Funct. Anal., 58, (1984), p. 225-253.

[C.] M. Chipot : Numerical analysis of oscillations in nonconvex problems. Numerische Mathematik, 59, (1991), p. 747-767.

[Ch.] M. Chipot : The appearence of microstructures in problems with incompatible wells and their numerical approach. Numerische Mathematik 83, No.3, (1999), p. 325-352 .

[C.C.] M. Chipot and C. Collins : Numerical approximation in variational problems with potential wells. SIAM J. of Numerical Analysis, 29, 4, (1993), p. $473-487$.

[Ko.] R. Kohn : The relationship between linear and nonlinear variational models of coherent phase transitions. In Proceedings of seventh Army Conference on applied Mathematics and Computing, West Point, June 1989.

[K.M.1] R. V. Kohn and S. Müller : Surface energy and microstructure in coherent phase transitions. Comm. Pure App. Math. 47, 4, (1994), p. 405-435.

[K.M.2] R. V. Kohn and S. Müller : Branching of twins near an austenite/twinned martensite interface. Phil. Mag. Ser. A 66 (1992) 697-715.

[P.] P. Pedregal : Parametrized measures and variational principles, Birkhäuser, 1997.

[W.] M. Winter : Lavrentiev phenomenon in microstructure theory. Electron. J. Differential Equations, No 6 (1996). 